\title{
Humanizing glycosylation pathways in eukaryotic expression systems
}

\begin{abstract}
Glycosylation represents the most widespread posttranslational modifications, found in a broad spectrum of natural and therapeutic recombinant proteins. It highly affects bioactivity, site-specificity, stability, solubility, immunogenicity, and serum half-life of glycoproteins. Numerous expression hosts including yeasts, insect cells, transgenic plants, and mammalian cells have been explored for synthesizing therapeutic glycoproteins. However, glycosylation profile of eukaryotic expression systems differs from human. Glycosylation strategies have been proposed for humanizing the glycosylation pathways in expression hosts which is the main theme of this review. Besides, we also highlighted the glycosylation potential of protozoan parasites by emphasizing on the mammalian-like glycosylation potential of Leishmania tarentolae known as Leishmania expression system.
\end{abstract}

Keyword: Glycosylation pattern; Glycoengineering; LEXSY; Eukaryotic expression systems 